\title{
Desafío en el diseño de MOOCs: incorporación de aspectos para la colaboración y la gamificación
}

\section{MOOC Design Challenges: Incorporating Collaboration and Gamification}

$$
\text { Aspects }
$$

\author{
Carina S. González \\ Universidad de La Laguna. España. \\ cjgonza@ull.edu.es \\ César A. Collazos \\ Universidad del Cauca. Colombia. \\ ccollazo@unicauca.edu.co \\ Roberto García \\ Universitat de Lleida. España. \\ roberto.garcia@udl.cat
}

\begin{abstract}
Resumen
En la actualidad, existe una gran cantidad de cursos MOOCs ofrecidos por diferentes proveedores, tales como MiriadaX, Coursera y edx, que se conforman a través de elementos independientes (charlas estilo TEDx, libros de texto digitales o programas de televisión educativos), y donde cada uno estos elementos ha sido diseñado y elaborado por un único profesor, como parte de una serie de vídeos con contenido digital, que se emitirá a un público de estudiantes que agradecerán dicho contenido audiovisual. Sin embargo, lo ideal es que los MOOCs impliquen procesos de colaboración en el que tanto profesores como alumnos, puedan participar en el diseño y de esta forma, utilizar de manera más eficaz estos cursos. En este sentido, nos encontramos ante el desafío de incorporar aspectos de colaboración en MOOCs, lo cual no es una tarea sencilla ya que implica el diseño y la organización de actividades que fomenten la colaboración real entre los participantes. Asimismo, son muy importantes los aspectos motivacionales para evitar el abandono de los cursos y favorecer el aprendizaje. En este punto, considerar algunas técnicas de gamificación para favorecer el aprendizaje puede resultar un factor determinante. Por ello, en este artículo se presenta una propuesta que incluye aspectos de colaboración y técnicas de gamificación en la planificación, diseño, implementación y uso de MOOCs. Además, se presenta una arquitectura tecnológica que permite el aprendizaje ubicuo basada en la Arquitectura de Formación y Aprendizaje (TLA).
\end{abstract}

\section{Palabras clave}

MOOCs, CSCL, uLearning, Gamificación, TLA, XAPI

\begin{abstract}
Nowadays, there is a great variety of Massive Online Open Courses (MOOCs) offered by different providers such as MiriadaX, Coursera, or edX. They are materialized using different elements (talks like TEDx, digital textbooks or educational TV programs), which are designed and developed by an instructor as part of a series of digital videos. Videos are then made available to the students that will access them. However, the ideal situation is that MOOCs motivate collaboration processes involving both teachers and students, who participate in the design of the courses so that they can more effectively use these courses. To achieve this, the challenge is to incorporate collaboration aspects into MOOCs. This is
\end{abstract}


not an easy task because it requires the design and organization of activities that motivate the collaboration among participants. Likewise, the motivational aspects are necessary to avoid courses dropout and favor learning. To this end, it is important to consider some gamification techniques that might be determinant to motivate learning. In this line, the proposal is the inclusion of collaboration and gamification techniques during MOOCs planning, design, implementation and use. This is integrated into a proposed technological architecture that facilitates ubiquitous learning, the Training and Learning Architecture (TLA).

\section{Key words}

MOOCs, CSCL, uLearning, Gamification, TLA, XAPI

\section{Introducción}

Es un hecho que la tecnología y la globalización han hecho que la educación superiorpueda ser más accesible, generando no solo un mayor número de oportunidades sinoalgunas incertidumbres. En este sentido, los cursos online masivos abiertos (MOOCs) son un fenómeno reciente y muy popular en la educación a distancia y el elearning, que han acercado aún más la formación en diversos niveles a la sociedad de la información.

Los MOOCs han sido aclamados por muchos como una solución a la falta de acceso a la educación en los países en desarrollo, dado que pueden proporcionar a un gran número de estudiantes de cualquier parte del mundo oportunidades para estudiar, siempre y cuando dichos estudiantes puedan tener acceso a través de Internet a dichos cursos (Liyanagunawardena, Williams y Adams, 2013). Si bien pueden ser considerados como una extensión de los enfoques de aprendizaje electrónico existente, los MOOCs ofrecen la oportunidad de repensar acerca de los nuevos modelos de educación superior en línea y gratuita, en dónde los cursos están abiertos a cualquier usuario de Internet.

Este modelo ha sido promovido por universidades de prestigio tales como Harvard, el Instituto de Tecnología de Massachusetts (MIT) o Stanford, y probablemente debido a ello, los MOOCs han disfrutado de una creciente presencia en el Internet en los últimos años. La iniciativa MOOC ha tenido un gran impacto, y ha sido consideradacomo uno de los mayores avances tecnológicos en el año2012 (Pappano, 2012). Muchas obras (por ejemplo, Daniel, 2012) mencionan la importancia de MOOCs y atribuyen su éxito a diferentes factores, entre ellos la masividad, la apertura, una filosofía conectivista, la accesibilidad, la flexibilidad y el convertirse en un fenómeno mundial.

McAuley, Stewart, Siemens y Cormier (2010) han explicado que los MOOCs ofrecen la conectividad de las redes sociales y una enorme variedad de materiales en línea gratis, pero con los beneficios adicionales de expertos en la materia que facilitan la asimilación de contenidos. Los MOOCs también permiten que los estudiantes puedan participar con otros estudiantes del mundo, y al mismo tiempo, que puedan organizan subgrupos específicos en función de sus objetivos e intereses de aprendizaje.

Las características que definen a este tipo de cursos MOOCs y su calidad varían mucho. Kinash (2013) ha identificado varias características que diferencian a los MOOCs de otras formas de educación a distancia, tal y como se muestra en la Tabla 1. 


\begin{tabular}{|c|c|}
\hline Característica & Descripción \\
\hline $\begin{array}{l}\text { Asignaturas o temas solos e } \\
\text { independientes }\end{array}$ & $\begin{array}{l}\text { Los MOOCs no están ligados formalmente a las } \\
\text { universidades. Suelen ser sobre temas independientes en } \\
\text { los que cualquier persona desde cualquier lugar puede } \\
\text { inscribirse }\end{array}$ \\
\hline Componente de red social & $\begin{array}{l}\text { La red social es un componente importante de un MOOC. } \\
\text { El diseño de muchosMOOCsse asemejan a una red social } \\
\text { tipo Facebook, en donde los estudiantes pueden hacer } \\
\text { amigos e interactuar el entre ellos. }\end{array}$ \\
\hline $\begin{array}{l}\text { Actividades de aprendizaje } \\
\text { sincrónicas y asincrónicas }\end{array}$ & $\begin{array}{l}\text { Los MOOCsa menudo equilibran actividades de } \\
\text { aprendizaje en tiempo real (sincrónico) y en cualquier } \\
\text { momento (asíncrono). Por ejemplo, habrá momentos en } \\
\text { que el profesor y los alumnos están en línea al mismo } \\
\text { tiempo charlando a través de voz o de texto. Otras } \\
\text { actividades están diseñadas para permitir que el estudiante } \\
\text { las realice en cualquier momento, de forma que se adapte } \\
\text { a su horario personal. }\end{array}$ \\
\hline Aprendizaje a tu "propio ritmo" & $\begin{array}{l}\text { A menudo hay suficientes materiales y ejercicios puestos } \\
\text { a disposición de los alumnos desde el momento en que } \\
\text { entran en el MOOC que son capaces de gestionar su } \\
\text { aprendizaje según su propio ritmo. Por lo general, estas } \\
\text { actividades no están vinculadas a los semestres } \\
\text { universitarios tradicionales. Algunos MOOCs permiten } \\
\text { que el estudiante pueda tomarse el tiempo tanto o tan poco } \\
\text { como lo que necesitan para que puedan ganar sus } \\
\text { certificados / créditos (cuando hayan completado } \\
\text { suficientes módulos o ganado suficientes créditos para } \\
\text { terminar el curso). }\end{array}$ \\
\hline $\begin{array}{l}\text { Diseño temporal } \\
\text { programado }\end{array}$ & $\begin{array}{l}\text { Mientras se proporcionan materiales suficientes para la } \\
\text { autogestión del tiempo, los MOOCsa menudo también } \\
\text { utilizan un diseño de liberación programada de } \\
\text { contenidos. Los educadores deben evitar abrumar a los } \\
\text { alumnos con excesivo contenido, y esto se hace } \\
\text { programando las fechas de lanzamiento de los mismos de } \\
\text { manera que el plan de estudios se revela a través del } \\
\text { tiempo. }\end{array}$ \\
\hline Aprendizaje adaptativo & $\begin{array}{l}\text { Muchos MOOCs aplican el concepto de aprendizaje } \\
\text { adaptativo. En este tipo de cursos, la dificultad de los } \\
\text { contenidos y el nivel de dificultad de los ejercicios se } \\
\text { ajustan al nivel del estudiante. Por ejemplo, si un } \\
\text { estudiante está errando en repetidas ocasiones sobre } \\
\text { determinadas áreas de contenido o habilidades } \\
\text { fundamentales; el sistema proporcionará automáticamente } \\
\text { las ayudas necesarias y ajustará el nivel de las actividades } \\
\text { al más acorde. }\end{array}$ \\
\hline
\end{tabular}

Desafío en el diseño de MOOCs: incorporación de aspectos para la colaboración y la gamificación. Carina S. González, César A. Collazos y Roberto García. 


\begin{tabular}{|l|l|}
\hline $\begin{array}{l}\text { Últimas características de diseño } \\
\text { tecnológicos habilitados }\end{array}$ & $\begin{array}{l}\text { Muchos MOOCs aprovechan las últimas tecnologías que } \\
\text { permiten o mejoran el aprendizaje. Por ejemplo, el } \\
\text { aprendizaje adaptativo es una característica de diseño de } \\
\text { tecnología de sistemas inteligentes que tienen algunos } \\
\text { MOOCs ya incorporan, analizando patrones de } \\
\text { comportamiento basados en técnicas de minería de datos. }\end{array}$ \\
\hline $\begin{array}{l}\text { Aprendizaje y evaluación entre } \\
\text { pares }\end{array}$ & $\begin{array}{l}\text { La mayoría de MOOCs están diseñados para utilizar la } \\
\text { evaluación en línea. Aquí los estudiantes completan sus } \\
\text { actividades de evaluación en línea y / o realizan la } \\
\text { presentación de ejercicios en línea, y muchos se entrenan } \\
\text { en la evaluación de pares, siendo ésta parte de su propia } \\
\text { evaluación. }\end{array}$ \\
\hline $\begin{array}{l}\text { Multimedia y aprendizaje } \\
\text { basado en juego }\end{array}$ & $\begin{array}{l}\text { Gran parte del contenido de los MOOCs es multimedia. } \\
\text { Los MOOCs son a menudo ricos en vídeo y en el } \\
\text { aprendizaje basado en el juego (puntos, insignias, etc.). }\end{array}$ \\
\hline $\begin{array}{l}\text { Contenido fraccionado en } \\
\text { unidades cortas }\end{array}$ & $\begin{array}{l}\text { El contenido en los MOOCssuele segmentarse en } \\
\text { unidades cortas. Por ejemplo, hay una serie de cinco } \\
\text { videos de 10 minutos de duración, intercalados con } \\
\text { actividades de aprendizaje y de evaluación. }\end{array}$ \\
\hline
\end{tabular}

Tabla 1. Las características que diferencian a losMOOCs de otras formas de educación a distancia (adaptado de Kinash, 2013)

Un estudio de un millón de usuarios de MOOCs publicados por la Universidad de Pennsylvania Escuela de Graduados en Educación (Lewir, 2013) encontró que, en promedio, la mitad de los estudiantes que se habían registrado a un curso jamás había visto una conferencia, y sólo alrededor del 4\% del total de los alumnos habían terminado los cursos en realidad. Por otra parte, una encuesta sobre 103 miembros del profesorado de MOOCs en Coursera produjo resultados interesantes (Kolowich y Newman, 2013). A pesar de que 79\% de los encuestados pensaba que losMOOCs valían la pena, sólo el $48 \%$ consideraba que su MOOC era menos riguroso académicamente que la versión en un aula tradicional del mismo curso, y sólo $28 \%$ creía que los estudiantes deberían recibir crédito institucional para completar su MOOC.

En encuestas realizadas por investigadores de la Universidad de Duke, el 95\% de los alumnos identificaba a la diversión y el disfrute como razones importantes para inscribirse en un MOOC antes de comenzar (Belanger y Thornton, 2013). Sin embargo, al término del curso MOOC, la mayoría de los estudiantes (87\%) atribuían su inscripción a un interés general en la materia. Por ello, creemos que se necesita más investigación para entender la motivación del alumno desde el inicio de un MOOC y los aspectos que mantienen la motivación durante el curso.

Zapata (2014) analiza el papel de los MOOCs en la crisis de la educación universitaria, presentando claves para el diseño instruccional y aprendizaje a través de los mismos desde el punto de vista de las teorías de aprendizaje y de la situación actual de la educación superior. Este mismo autor destaca que este tipo de cursos "deberían ofrecer oportunidades para la interacción entre el profesor y los alumnos y entre los propios alumnos, una opción de la cual carecen muchos MOOC”. Además, avanza en otras características que se deberían incluir, tales como las analíticas de aprendizaje y la personalización. 
Como tendencias en los MOOCs podemos notar que los proveedores MOOC se han movido hacia un modelo de cursos en dónde los estudiantes pueden realizar los cursos a propio ritmo, lo que significa que los cursos están siempre abiertos y los usuarios pueden completar un curso en diferentes sesiones. De esta forma, si un estudiante no es capaz de terminar el curso en la sesión actual, siempre pueden pasar a la siguiente sesión sin perder el curso. También están apareciendo MOOCs no solo destinados a la educación universitaria, sino a los estudiantes de secundaria, con la intención de ayudar en la preparación para el ingreso en la universidad, ofreciendo una muestra de diferentes especialidades a través de cursos de iniciación y ayuda a la preparación de exámenes de ingreso (Shah, 2015). Podemos ver también una generación de MOOCs diferente: en la primera la idea era simplemente crear un catálogo de cursos gratuitos y la segunda está orientada a que los estudiantes obtengan resultados más prácticos y obtengan las credenciales asociadas, por ejemplo a través de Nanodegrees (Udacity), especializaciones (Coursera) o un programa o serie de cursos (XSeries Program de edX). La idea de esta generación actual de MOOCs es ofrecer una propuesta más clara de valor para el estudiante. Por ello, para 2016 uno de los desafíos es encontrar y potenciar los componentes críticos de la experiencia de aprendizaje en los MOOCs (Shah, 2015).

Por lo tanto, nos preguntamos ¿qué está pasando con los MOOCs?, ¿cuáles son los problemas reales que les afectan?, ¿cómo pueden ser reorganizados para cumplir con su promesa inicial? y, ¿cómo podemos potenciar la experiencia de aprendizaje en los mismos?. Estas preguntas nos han servido como punto de partida para el análisis de cómo los elementos de colaboración y gamificación podrían integrarse en MOOCs para que sean más eficaces.

Actualmente, las interfaces a través del cual se realizan los MOOCs en las plataformas masivas no permiten a los estudiantes a conectarse e interactuar de forma sincrónica, colaborar o ver su progreso. El principal problema con MOOCs es su falta de capacidad para la colaboración de los alumnos a través de una interfaz centrada en el estudiante. En este trabajo se presenta un modelo que permita tanto a los profesores y como a los alumnos diseñar el aprendizaje de manera cooperativa, implementar y utilizar MOOCs. Eso permitiría que los profesores compartan experiencias e información específica sobre la materia que se estudia, lo que implica la construcción del conocimiento colectivo. El mayor reto de la educación en la sociedad del conocimiento no consiste en ayudar de manera efectiva a que los estudiantes adquieran conocimientos y habilidades, sino en ayudar a aprender a gestionar y trabajar creativamente con las ideas y contribuir a la creación de nuevos conocimientos.

En este artículo se presenta una versión extendida del modelo, parcialmente presentado en Collazos, González y García (2014), denominado "Computer Supported Collaborative MOOCs: CSCM", en donde se presenta la propuesta de la arquitectura tecnológica del modelo basada en TLA. En el presente artículo, se profundiza en el análisis del los desafíos en el diseño de MOOCs relacionados con la incorporación de aspectos relacionados con la colaboración y la gamificación. Por ello, se realiza un análisis de las ideas provenientes de la literatura actual sobre el tratamiento e incorporación los aspectos y métodos colaborativos, pedagógicos y tecnológicos en el

Desafío en el diseño de MOOCs: incorporación de aspectos para la colaboración y la gamificación. Carina S. González, César A. Collazos y Roberto García. 
diseño de alternativas de futuro de los MOOCs. El artículo se organiza de la siguiente manera: la sección 2presenta los aspectos relacionados al aprendizaje colaborativo, la sección 3 analiza los principales trabajos relacionados con la temática, y luego, se describe la propuesta del modelo de colaboración en MOOCs y su aplicabilidad. Finalmente, se presentan las conclusiones y el trabajo futuro.

\section{2.- Aspectos de colaboración}

El aprendizaje colaborativo tiene muchas formas y definiciones, pero la mayoría de los enfoques de colaboración involucra equipos pequeños, heterogéneos, por lo general de cuatro o cinco miembros, trabajando juntos para llevar a cabo una tarea de grupo, en el que cada miembro es individualmente responsable de parte de un resultado que no se puede lograr a menos que todos ellos cooperan. En otras palabras, los miembros del grupo son positivamente interdependientes. Un claro ejemplo de la interdependencia es la relación entre el lenguaje de minorías y los alumnos de lengua mayoritaria en programas de inmersión de dos vías.

En una colaboración exitosa la sinergia es mayor, y los resultados son mejores que la suma de los resultados individuales de sus partes. Para que los grupos de colaboración sean eficaces, los miembros deben participar en actividades de trabajo en equipo y otras tareas que tienen que ver explícitamente con el desarrollo de las habilidades sociales necesarias de un trabajo en equipo. Los miembros también deben participar en actividades de procesamiento de grupo en el que se discuten la eficacia del trabajo conjunto y donde las habilidades interpersonales que influyen en el correcto funcionamiento del grupo.

El aprendizaje colaborativo es una teoría educativa que involucra a dos o más personas que trabajan juntos para aprender algo; una vieja idea de que se está convirtiendo en parte de muchos entornos digitales educativos. Se basa en la premisa general de que los grupos de alumnos pueden aprender más de lo que lo haría si aprendieron por su cuenta, aprendiendo unos de otros mediante el intercambio y la interacción social. Según los defensores de esta teoría, el intercambio de forma activa, el debate y la negociación de las ideas con sus compañeros aumenta el interés los alumnos en el aprendizaje. Aún más relevante, mediante la participación en el debate y asunción de la responsabilidad de su aprendizaje, se anima a los estudiantes a convertirse en pensadores críticos (Totten, Sills, Digby y Russ, 1991).

El aprendizaje colaborativo es un fenómeno complejo que se está estudiando en diferentes niveles de análisis y perspectivas teóricas y metodológicas. Numerosos investigadores han informado de que los estudiantes que trabajan en pequeños grupos tienden a aprender más de lo que se enseña. Además, conservan la información más tiempo y también parecen más satisfechos con sus clases (Johnson y Johnson, 1986).

Pero, poner simplemente un grupo de alumnos a trabajar en una tarea no garantiza la colaboración real. También es necesario establecer actividades específicas que generan este tipo de colaboración. Tales actividades deben ser cuidadosamente diseñadas, 
planificadas y organizadas, estar bien estructuradas, y tener subtareas claramente definidas relacionadas a los objetivos educativos que se deseen alcanzar.

Muchos autores han descrito los principales elementos de aprendizaje colaborativo. Johnson, Johnson y Smith (2006) proponen cinco principios, que son la interdependencia positiva, la interacción cara a cara, la igualdad de participación, la responsabilidad individual y las habilidades sociales. Soller (2001) identifica elementos tales como la participación activa de los alumnos, establecer y mantener un entendimiento común, el pensamiento crítico, la evaluación progresiva y la ayuda mutua.

Aunque estamos de acuerdo con estas propuestas, nuestra posición es más cercana a la de autores como Kagan (1990) y Wenger (2009), que consideraban que una buena cooperación puede lograr sobre la base de tres elementos, la interdependencia positiva específicamente, la igualdad de participación y la responsabilidad individual. En nuestra opinión, la presencia de, al menos, estos tres elementos hará que un grupo funcione mejor y experimenten menos problemas en el cumplimiento de su objetivo común (la solución de un problema, el diseño de un artefacto, etc.). Por otra parte, el aprendizaje colaborativo asistido por ordenador (CSCL) es una rama emergente de las ciencias del aprendizaje relacionados con el estudio de cómo las personas pueden aprender juntos con la ayuda de los ordenadores. Esta simple afirmación esconde una considerable dificultad ya que la interacción entre el aprendizaje y la tecnología es una cuestión compleja. La inclusión de la colaboración, la mediación informática y la educación a distancia ha problematizado la noción misma de aprendizaje en línea y ha puesto en duda sobre cómo debía ser estudiado (Stahl, Koschmann y Suthers, 2006). Teniendo todo esto en mente, nuestra propuesta (que se describe en la sección 4 de este documento) pretende incluir aspectos de colaboración en los MOOCs sdesde la perspectiva del CSCL.

\section{3.- Trabajo Relacionados}

Diferentes ideologías han dirigido los MOOCs en direcciones pedagógicas diferentes, dando lugar, principalmente a diferentes tipos de cursos.Por su relevancia, en este artículo veremos dos tipos: los cMOOCs y los xMOOCs. Los MOOCs conectivistas (cMOOCs), que se basan en una teoría conectivista del aprendizaje con redes desarrolladas informalmente; y MOOCs basados en contenido (xMOOCs), que siguen un enfoque más conductista y se basan en un modelo más individualista del aprendizaje, mediante el cual alumnos individuales, distribuidos geográficamente, siguen una secuencia de vídeos formativos e interactúan de forma solitaria con cuestionarios entrelazados. En muchos sentidos, este es el mismo proceso de aprendizaje centrado en contenidos que los educadores han tenido durante muchas décadas, que ha sido sometido a debate y que no se ha podido resolver.

Los cMOOCs enfatizan el aprendizaje conectado, colaborativo, y están construidos alrededor de un grupo de "individuos" de ideas afines que están relativamente libres de restricciones institucionales. Estos cursos proporcionan una plataforma para explorar nuevas pedagogías más allá de las aulas tradicionales y, como tal, tienden a existir en el sector más radical de la educación superior. El modelo de instrucción de los xMOOC,

Desafío en el diseño de MOOCs: incorporación de aspectos para la colaboración y la gamificación. 
por el contrario, es esencialmente una extensión de los modelos pedagógicos aplicados dentro de las propias instituciones. Está dominado posiblemente por los métodos de enseñanza centrados en la planificación formal de contenidos, incluyendo presentaciones de vídeo, cuestionarios y pruebas cortas (Downes, 2013).

Con los cMOOCs, se espera que los alumnos puedan hacer una contribución activa a través de diferentes plataformas digitales. Tales contribuciones (por ejemplo, en forma de tweets, entradas de blog, etc.) son moderadas por los supervisores del curso y entregadas a cada participante del curso en un boletín diario o por correo electrónico. Además, cMOOCs no son generalmente financiados o patrocinados por organismos de educación superior. En cambio, sí participan personas que tienen un interés en un área de conocimiento en particular. Los organizadores dedican tiempo a la creación de un marco educativo en el que estudiantes de todo el mundo pueden compartir, conectar, participar y colaborar para comprender contenidos y al mismo tiempo, ampliar su red de contactos profesionales y personales. Además, los cMOOCs están abiertos y son adaptables, son sensibles a las necesidades de los alumnos y pueden ofrecer un ambiente de aprendizaje a medida.

Los xMOOCs han sido criticados por la adopción de un modelo de transmisión de conocimientos. En esencia, se considera siguen el modelo de la enseñanza centrada en el profesor tradicional, enriquecida con tecnología (Larry, 2012). Ofrecen una experiencia individualizada permitiendo a los estudiantes tomar rutas alternativas a través del material que ofrecen y proveen de retroalimentación, normalmente automática. Sin embargo, no proporcionan una experiencia de aprendizaje social o atención personal. Coursera, por ejemplo, deja claro que el diseño depende de cada institución, dentro de las pautas generales dadas por la plataforma. Por lo tanto, es probable entonces que muy pocas instituciones tengan suficiente personal con conocimiento práctico importante de la pedagogía en línea para atender con calidad este tipo de cursos.

Por el contrario, los cMOOCs ofrecen grandes oportunidades para las formas no tradicionales de los enfoques de enseñanza y la pedagogía centrada en el alumno, en dónde los alumnos aprenden el uno del otro. Las comunidades en línea que se generan en este tipo de cursos distribuyen el aprendizaje de una manera que rara vez se da en las aulas tradicionales dentro de las universidades. Por ejemplo, instituciones como el MIT y la Universidad de Edimburgo han utilizado los MOOCs como una aventura experimental para participar en modelos pedagógicos emergentes, en donde apoyo entre los compañeros y la evaluación por pares es fundamental para el aprendizaje.

Como variantes de MOOCs podemos mencionar los siguientes modelos híbridos (Coughlan, 2014):

- Big Open Online Course (BOOC): sigue un formato híbrido ya que combina el aprendizaje distribuido (cMOOC) con retroalimentación personalizada (xMOOC) y se basa en la teoría de la cognición situada centrándose en el aprendizaje contextual (Hickey, 2013).

- Distributed Open Collaborative Course (DOCC): sigue la pedagogía híbrida que incluye vídeosconferencias a cargo de expertos (xMOOC) y reconoce que el 
conocimiento se distribuye en red (cMOOC). Los materiales de aprendizaje, lecturas y tareas se distribuyen y se basa en el plan de estudios, la rúbrica de clasificación y la personalización.

- Little Open Online Course (LOOC): El modelo sigue el formato xMOOC basado en vídeos elaborados por un profesor, podcasts y la participación a través de discusiones.

- Massive Open Online Research (MOOR): sigue el modelo xMOOC que incluye videos por parte de la instructores. Los materiales del curso incluyen un e-book escrito por los instructores del curso que incorpora ejercicios y la resolución de problemas. Este modelo incorpora la Zona de Desarrollo Próximo (ZDP) de Vygotsky. Así, cada capítulo del texto se basa en los conceptos e ideas básicas para introducir temas complejos y evalúa el dominio de conceptos de aprendizaje a través de desafíos y las tareas. El curso incluye un apoyo $24 \times 7$ por parte de los instructores.

- Small Private Online Course (SPOC): se inspira en los xMOOC e incluye vídeos del instructor, tareas interactivas y discusión en grupos. Sigue el modelo de "aula invertida o flipped classroom" (Coughlan, 2013). Los estudiantes online se matriculan como estudiantes presenciales tradicionales, que a su vez ejercen de mentores.

- Synchronous Massive Open Online Courses (SMOC): sigue el modelo xMOOC, en donde los materiales de aprendizaje incluyen recursos web gratuitos y lecturas en ñínea. Las conferencias en directo se utilizan para involucrar a los estudiantes y la participación de los alumnos se fomenta a través de la participación en foros de discusión. Las clases se dividen en grupos pequeños y controlados por los tutores que son los ex alumnos del curso. Tener los mismos estudiantes en el mismo grupo a lo largo del semestre ayudar a crear un sentido de comunidad y promueve la interacción constante entre los estudiantes.

Actualmente, existen numerosas plataformas de MOOCs, citaremos algunas de las más populares:

- edx (https://www.edX.org/)

- Coursera (https://www.coursera.org/)

- Udacity (https://www.udacity.com/)

- Udemy (https://www.udemy.com/)

- P2PU (https://p2pu.org/en/)

- Khan Academy (https://www.khanacademy.org/)

- Course Builder (https://code.google.com/p/course-builder/wiki/ListOfCourses)

- SlideWiki (http://slidewiki.org)

- UniMooc (http://iei.ua.es/mooc-emprendimiento/)

- RedUnX (http://www.colmenia.org/)

- MiriadaX (https://www.miriadax.net)

Como trabajos relacionados, mencionaremos también a una serie de iniciativas orientadas a la inclusión de aspectos de colaboración en MOOCs, tales como el curso distribuido de colaboración abierto (DOCC), en donde, en lugar de crear un curso con 
planes de clase completos, lecturas y tareas, como sucedería en el caso de un MOOC, el prototipo DOCC ofrece instructores con una discusión grabada en vídeo sobre un tema y anima a los participantes a compartir las tareas y otros materiales de los cursos entre ellos. En otras palabras, las piezas básicas de contenidos de aprendizaje están en el DOCC, pero cada elemento está fuertemente personalizado por el instructor para que coincida con las condiciones en su salón de clases. Este parece ser un modelo que podría ser más atractivo para los profesores. Les da una "fuente primaria" y un tema, pero les anima a construir un campo a su alrededor en la forma en que ellos piensan que será mejor para sus alumnos (Blake, 2013).

NovoEd es un proveedor de cursos MOOC creado por el profesor de la Universidad de Stanford AminSaberi y su estudiante de doctorado Farnaz Ronaghi. Originalmente llamado Venture Lab, NovoEd tiene la intención de fomentar el trabajo más colaborativo en pequeños grupos, un rasgo que lo diferencia de otras plataformas MOOC actuales.

Blom, Verma, Li, Skevi y Dillenbourg (2013) han informado en dos estudios que desafían el modelo individualista del aprendizaje basado en MOOC, mediante la incrustación del uso de MOOCs en el contexto de grupos de estudio con ubicación compartida. La capacidad de hacer una pausa en una conferencia y discutir su contenido con compañeros crea oportunidades de aprendizaje. Además, los autores han estado investigando la mejor manera de configurar este tipo de grupos de estudio con diferentes herramientas.

El principal problema de las iniciativas en cuestión es que no garantizan la colaboración real. Estableciendo simplemente un grupo de personas para trabajar en una tarea no significa que vayan a colaborar. También es necesario organizar actividades que requieren la participación y la colaboración (Collazos et al., 2007).

Por tanto, es importante tener en cuenta qué elementos deben incluirse en los MOOCs que proporcionen los mecanismos necesarios para la colaboración y la comunicación efectiva en un entorno en línea. El aprendizaje colaborativo y la comunicación son una parte esencial de losMOOCs, ya que permiten a los estudiantes a desarrollar las habilidades necesarias, incluyendo la cooperación y la comunicación efectiva en un ambiente en línea.

Por otra parte, el aprendizaje colaborativo y la comunicación crean un marco para la construcción y / o el intercambio de conocimientos en una materia determinada, que puede conducir a un aprendizaje más profundo y significativo (Morrison, 2013). Los MOOCs podrían descentrar el papel del profesor y llevar a la interacción colaborativa entre los estudiantes, quienes de este modo tendrían más poder y responsabilidad en su aprendizaje. Nuestro modelo incluye elementos para que este aprendizaje colaborativo en los cursos MOOCs se desarrolle de forma efectiva.

\section{4.- Modelo de colaboración}

Los entornos en línea actuales deben incorporar tecnologías colaborativas acordes con los avances de Internet, tales como han madurado las herramientas distribuidas de desarrollo de software (por ejemplo, Github, Bugzilla, CruiseControl), herramientas de

Desafío en el diseño de MOOCs: incorporación de aspectos para la colaboración y la gamificación. 
comunicación (por ejemplo, los wikis), herramientas que permiten colaboraciones virtuales entre una amplia gama de comunidades de desarrollo software, que han llegado a abarcar diferentes zonas horarias y traspasar los límites de la organización, apoyando diversos modelos de negocio y cambiando radicalmente cómo se desarrolla, prueba y evoluciona el software. La lección clave aquí es que la infraestructura digital ha sido transformadora, no sólo porque ha permitido a los desarrolladores acceder a contenidos de manera más flexible, sino también porque ha creado nuevas formas más potentes de colaboración. Vemos entonces como la colaboración es en un aspecto crucial de este nuevo modo interactivo.

Nuestra propuesta es una extensión del aprendizaje colaborativo apoyado por ordenador (CSCL) (Collazos et al., 2014), en dónde se han incluido diferentes aspectos para establecer una colaboración real entre los participantes. La Figura 1 muestra el modelo que hemos diseñado e implementado. El objetivo principal de nuestra propuesta es integrar los aspectos de colaboración y de aprendizaje ubicuo, que se da a través de los siguientes elementos principales:(I) Los profesores; (II) Un entorno de colaboración; (III) Los recursos de estudio (contenidos, actividades de colaboración); (IV) Un repositorio de objetos de aprendizaje; (V) Una plataforma tecnológica (sistemas de gestión de aprendizaje, entornos de aprendizaje virtual); (VI) Servicios de acceso y (VII) Estudiantes. La primera parte del modelo incluye un ecosistema en el que los profesores establecen una comunidad para compartir objetivos. Funciona como una red social en la que diferentes profesores deciden participar en la creación de MOOCs. Una vez que se ha tomado esa decisión, la idea es formar vínculos entre los profesores con el mismo interés y conocimiento en ciertos temas. El mecanismo tradicional para generar contenido en un MOOC involucra a una persona responsable de diseño de materiales educativos. En nuestro modelo, varios profesores, con diferentes experiencias relacionadas con el mismo tema, diseñan los materiales, haciendo que el contenido que crean sea más realista. Nuestro modelo permite la discusión sobre los contenidos, que se determinará de forma colaborativa. Por lo tanto, el modelo anima a la interacción del profesor-profesor en los foros de discusión. Es importante señalar, sin embargo, que se ha encontrado que simplemente el acceso a una herramienta de comunicación (por ejemplo, un wiki, un foro público) no es suficiente para generar esta creación colaborativa de cursos. Ofrecer directrices y pautas para ayudar a los equipos trabajan juntos podría ser la clave. Por ello, se ofrece una guía con recomendaciones sobre el diseño de cursos en colaboración.

Johnson y Johnson (1986) mencionan que un proceso de aprendizaje cooperativo se compone típicamente de varias tareas que deben llevarse a cabo por el facilitador/profesor y por el grupo de aprendices. Los autores afirman que las diferentes actividades (por ejemplo, especificar el tamaño del grupo, proporcionar ayuda, etc.) se producen entre los miembros del grupo durante una tarea de colaboración. Hemos clasificado estas actividades en tres tipos, según el momento en que se llevan a cabo, es decir: pre-proceso, en proceso y post-proceso. Las tareas previas a los procesos de aprendizaje colaborativo son principalmente las actividades de coordinación y definición de estrategia, mientras que en las tareas de post-proceso se trabaja principalmente sobre las actividades de evaluación. Tanto las tareas de pre-proceso y post-proceso se realizan en su totalidad por el facilitador/profesor. Por otra parte, las 
tareas correspondientes a la fase "en proceso" son realizadas en gran parte por los miembros del grupo. Es aquí donde las interacciones cooperativas del proceso de trabajo se llevan a cabo. Las diferentes actividades y la fase a la que pertenece cada uno de ellas son las siguientes:

- Diseño de contenidos y establecer los objetivos principales a llevar a cabo por grupos de cooperantes (pre-proceso).

- Especificar el tamaño del grupo. El tamaño del grupo sugerido es de hasta seis personas, dependiendo de la naturaleza de la tarea y el tiempo disponible (preproceso).

- Formar grupos. Asignar a estudiantes a los grupos o permitir formar grupos libremente (pre-proceso).

- Organizar el espacio para la actividad de aprendizaje cooperativo. El facilitador debe ser "accesible" para todos los grupos, y los miembros de cada grupo deben ser capaces de estar juntos de forma separada, sin los miembros de otros grupos (pre-proceso).

- Distribuir los materiales de instrucción. Esto se puede realizar de varias maneras (pre-proceso).

- Establecer funciones a los miembros del grupo, tales como presentador, facilitador, registrador, ejecutor, observador, etc. (pre-proceso).

- Especificar las reglas del juego (pre-proceso).

- Aplicar estrategias para el aprendizaje colaborativo, como la interdependencia positiva en relación a la meta, la motivación y el apoyo entre pares.

- Crear un producto relacionado con un sistema de meta, donde las recompensas se basen en los resultados individuales y del grupo (definido en la fase previa al proceso, pero evaluado en la fase "en proceso").

- Organizar la cooperación intra-grupo, es decir, definir las estrategias de colaboración para ser utilizados por los miembros del grupo (pre-proceso, la materialización de las estrategias de cooperación se produce en la fase "en proceso").

- Definir / revisar los criterios de éxito, explicar pautas, límites y roles (preproceso, en proceso y post-proceso). Los criterios de éxito se deben definir en el comienzo de la actividad. Posteriormente, deben ser revisados durante la actividad para comprobar si se está logrando el objetivo común, y luego otra vez después de la actividad para comprobar si realmente se logró.

- Determinar el comportamiento deseado (pre-proceso, la materialización de la conducta deseada se produce en la fase en proceso).

- Supervisar a los alumnos, verificar, por ejemplo, que el comportamiento deseado se materializa en realidad (en proceso).

- Proporcionar ayuda a petición (en proceso). Esta asistencia debe ser proporcionada para todo el grupo, por el facilitador o por sus compañeros.

- Intervenir cuando los grupos tienen problemas relacionados con la colaboración (en proceso).

- Llevar la actividad a una conclusión (post-proceso).

- Evaluar la calidad del aprendizaje logrado (post-proceso). 
- Animar a los estudiantes a evaluar cómo ha trabajado el grupo en conjunto (final de la fase "en proceso").

- Proporcionar y fomentar la retroalimentación. Discutir cómo se podrían mejorar las actividades (final de la fase "en proceso").

La tabla 2 resume las actividades involucradas en un proceso de aprendizaje cooperativo.

\begin{tabular}{|c|c|c|}
\hline Pre proceso & En proceso & Post proceso \\
\hline Diseñar los contenidos & $\begin{array}{l}\text { Aplicar estrategias } \\
\text { (interdependencia positiva de la } \\
\text { meta, motivación entre pares, } \\
\text { ayudar a aprender, etc.) }\end{array}$ & $\begin{array}{l}\text { Inspeccionar los criterios } \\
\text { de éxito }\end{array}$ \\
\hline $\begin{array}{l}\text { Especificarel tamaño de los } \\
\text { grupos }\end{array}$ & Cooperación intra-grupo & $\begin{array}{l}\text { Presentar el cierre de la } \\
\text { actividad }\end{array}$ \\
\hline Organizar los grupos & Sondear los criterios de éxito & $\begin{array}{l}\text { Evaluar la calidad del } \\
\text { aprendizaje }\end{array}$ \\
\hline Configurar el espacio & Escuchar & \\
\hline Distribuir el material & $\begin{array}{l}\text { Proporcionar ayuda (de facilitador y } \\
\text { de los compañeros) }\end{array}$ & \\
\hline Diseñar los documentos & Intervenir en caso de problemas & \\
\hline Especificar las reglas de juego & Revisión del grupo & \\
\hline Definir los criterios de éxito & Realimentación & \\
\hline $\begin{array}{l}\text { Determinar el comportamiento } \\
\text { deseado }\end{array}$ & & \\
\hline
\end{tabular}

Tabla 2. Las actividades en un proceso de aprendizaje cooperativo.

La parte pedagógica de nuestra propuesta incluye un modelo conceptual basado en los principios de diseño instruccional (Reigeluth, 2012) y los perfiles de usuario incluidos, los aspectos constructivistas para promover la participación de los alumnos activos en el proceso de enseñanza y aprendizaje, trabajando además habilidades como la colaboración, la comunicación y el trabajo en equipo.

En nuestro modelo, los profesores son los encargados de generar y desarrollar los contenidos y las actividades a través de un entorno de colaboración. Mientras que en el modelo tradicional este proceso es lineal, en nuestra propuesta tiene una estructura espiral en la que los ciclos de exploración, el aprendizaje y la creación de nuevos conocimientos se reiteran en los niveles más altos. En el proceso de generación de contenidos, los profesores pueden explorar los contenidos elaborados por otros colegas e investigadores / formadores y alterar objetos de conocimiento compartidos, permitiendo diferentes enfoques de las prácticas educativas. Como un mecanismo de apoyo a la creación de contenido, hemos desarrollado un marco de orientaciones y un software como guía esta creación colaborativa. 
Dicho marco tiene por objeto proporcionar a los profesores la orientación de las actividades de aprendizaje colaborativas y la definición de las tareas que se requieren para impulsar la cooperación real entre los participantes.

\section{5.- Modelo de gamificación}

Como han mencionado algunos autores como Yuan y Powell (2013), la motivación del alumno es un aspecto clave de MOOCs. ¿Por qué la gran mayoría de los alumnos que se inscriben en los cursos MOOCs nunca los terminan?. Una respuesta posible, muy simple, sería que los MOOCs no son lo suficientemente interactivos y atractivos. Sobre esa base, la gamificación (Zichermann y Cunningham, 2011) de los cursos MOOCs podría ser un tema importante a considerar y estudiar. La gamificación normalmente consiste en aplicar elementos de diseño de videojuegos a aplicaciones que no son lúdicas, para hacerlas más divertidas y atractivas, tales como crear tareas de aprendizaje en forma de juegos en los cursos (Romero\&Usart, 2013).

Kevin Werbach (2012) identifica tres categorías de elementos de juego, específicamente dinámica, mecánica y componentes. La dinámica del juego es la estructura implícita de un juego y determina cómo los jugadores interactúan en tiempo de ejecución. Se componen de niveles de desbloqueo, el compromiso social y la programación de recompensas. Las mecánicas del juego son los procesos que determinan cómo se desarrolla el juego, cómo los jugadores pueden y no pueden tratar de lograr sus metas (desafíos), y lo que sucede cuando tratan de alcanzarlos. Esta categoría comprende sistemas de puntos, niveles, el acceso y el poder y las tablas de clasificación. Por último, los componentes del juego son implementaciones específicas de la dinámica y la mecánica, e incluyen los avatares, las insignias, los puntos, las colecciones, las clasificaciones, los niveles, los equipos y los bienes virtuales.

Muchas las técnicas utilizadas en la gamificación de procesos pueden convertirse en estrategias útiles para ser aplicadas en las actividades de aprendizaje potenciando la motivación de los estudiantes hacia el aprendizaje (González y Mora, 2015). Por ello, siguiendo los elementos de juego descritos anteriormente, nuestra plataforma implementa algunas técnicas de gamificación integradas en el proceso de enseñanzaaprendizaje con el objetivo de aumentar y mantener la motivación de los estudiantes hacia el proceso seguido en los MOOCs (Borras, Martínez \& Fidalgo, 2014).

De esta forma, nuestro modelo cuenta con algunos componentes populares, tales como puntos, escudos y tablas de clasificación, comúnmente conocida como PBL (puntos, insignias y tablas de clasificación). Además, incorpora estrategias gamificación para actividades educativas, como la experimentación repetitiva, el incremento progresivo en el nivel dificultad de la tarea, la división de tareas en subtareas (más cortas y más simples), el diseño de diferentes rutas hacia el éxito, y la incorporación de las recompensas y el reconocimiento social (González, Mora y Toledo, 2014). También se propone integrar a los cursos MOOCs las actividades realizadas en las redes sociales utilizando métricas que midan la participación en dichas redes sobre temáticas relacionadas a los cursos, y de esta forma se impulsa el componente social del aprendizaje (De-Marcos et al, 2014). Por último, destacar que nuestra propuesta de la 
gamificación del aprendizaje colaborativo en MOOCs se basa en explotar la dimensión social del aprendizaje, en donde el aprendizaje se construye en una comunidad.

En nuestro modelo consideraremos además siguientes mecánicas de gamificación por estar incluidas en la mayoría de MOOCs y ser las más efectivas a la hora de generar "enganche" en los cursos (Chang y Wei, 2015): bienes virtuales, puntos rescatables, tablas de clasificación de equipo, trofeos e insignias, clasificación por parejas, retroalimentación por emoticones, puntos de comprobación y puntos de habilidad.

\section{6.- Modelo tecnológico}

Con respecto a su plataforma tecnológica, nuestro modelo incluye una arquitectura multiplataforma orientada a servicios para la gestión del conocimiento (Steenkamp\& González, 2011), que incorpora el aprendizaje móvil, multimedia, componentes de videojuegos, elementos de e-learning y servicios de t-learning. Por tanto, es un modelo multiplataforma, que sigue los principios de interoperabilidad y escalabilidad, capaz de generar y soportar nuevos componentes, que ofrece la opción de usar diferentes tipos de dispositivos, lo que permite un acceso ubicuo a la información, que por lo tanto se puede visualizar fácilmente, recuperada y utilizada (Cruz-Benito et al, 2015).

Nuestro modelo utiliza la arquitectura de Formación y Aprendizaje (TLA) (Regan, 2013). El TLA es el nombre dado a la siguiente generación Object Reference Model (SCORM) para compartir contenido (Poltrack, Hruska, Johnson y Haag, 2012) y del proyecto Tin Can. El TLA es una solución de interoperabilidad que comprende:

- La experiencia de seguimiento, para el seguimiento de las experiencias de aprendizaje de diferentes contextos, tales como cursos formales, simulaciones, aprendizaje informal en los sitios web y vídeos, juegos e interacciones enredes sociales. Este componente contiene las especificaciones de servicios web (Experience API (XAPI)) y de software (un repositorio de aprendizaje (LRS)), que pueden ser utilizados para rastrear los datos del alumno en base a las interacciones con las distintas experiencias de aprendizaje y para hacer que los datos almacenados disponibles para su uso en otro sistema.

- La intermediación y comprensión de contenidos, en la descripción, el descubrimiento y la entrega de contenido. Este componente cuenta con las especificaciones y el software para la gestión de contenido que permite apoyar el aprendizaje justo a tiempo y permitir la selección de la siguiente actividad. También incluye componentes inteligentes y contenido legible por los ordenadores para el recomendar y brindar la próxima experiencia de aprendizaje a un usuario y compartir contenido apropiado a su contexto y su dispositivo.

- Perfiles de aprendices, para perfiles de usuario. Este componente cuenta con especificaciones y software para facilitar el acceso a datos sobre los estudiantes, como sus preferencias y su historial. Permite la personalización de las experiencias de aprendizaje, teniendo en cuenta el estilo y la experiencia específica de cada alumno dentro de un sujeto. Los datos en que se basan los 
perfiles se pueden adquirir de muchas fuentes, como el seguimiento de experiencias e información dada por el alumno cuando se registró en el sistema.

- Redes de competencias, para la definición de objetivos y competencias. Este componente cuenta con especificaciones y software para relacionar los objetivos de aprendizaje y las definiciones de competencias con el contenido y los sistemas a utilizar.

Cada componente se concibe como un conjunto de una o más definiciones de servicios web estandarizados y software de código abierto asociado, que implementan los servicios web. En este punto, es importante tener en cuenta los estándares de e-learning para el seguimiento de la interoperabilidad de datos; en nuestro caso, la xAPI, anteriormente conocido como el Tin CanAPI. La xAPI es una extensión de los flujos de actividad (http://activitystrea.ms), especificación creada por Google, Facebook, Microsoft e IBM, entre otros, para capturar la actividad en las redes sociales. En el modelo de datos xAPI básica, la actividad del usuario se almacena en forma de declaraciones cuyo formato se basa en flujos de actividad. Sin embargo, el modelo de datos XAPI va más allá, y realiza el seguimiento de todos los aspectos de la experiencia de aprendizaje. Así, la xAPI parte de la iniciativa del TLA y presenta un modelo de datos flexible, añadiendo la posibilidad de compartir datos de seguimiento entre los diferentes sistemas (del Blanco et al., 2013). Se trata de una especificación de código abierto, con más de 100 adoptantes, proyectos y empresas participan actualmente en su desarrollo. La versión 1.0 se puso a disposición en abril de 2013. La especificación aumenta la interoperabilidad entre los enfoques de detección que pueden alimentar a la analítica de aprendizaje funciones (Corbi y Burgos, 2014). Es por lo tanto muy adecuado a las necesidades de las herramientas de análisis de aprendizaje (del Blanco et al., 2013). 


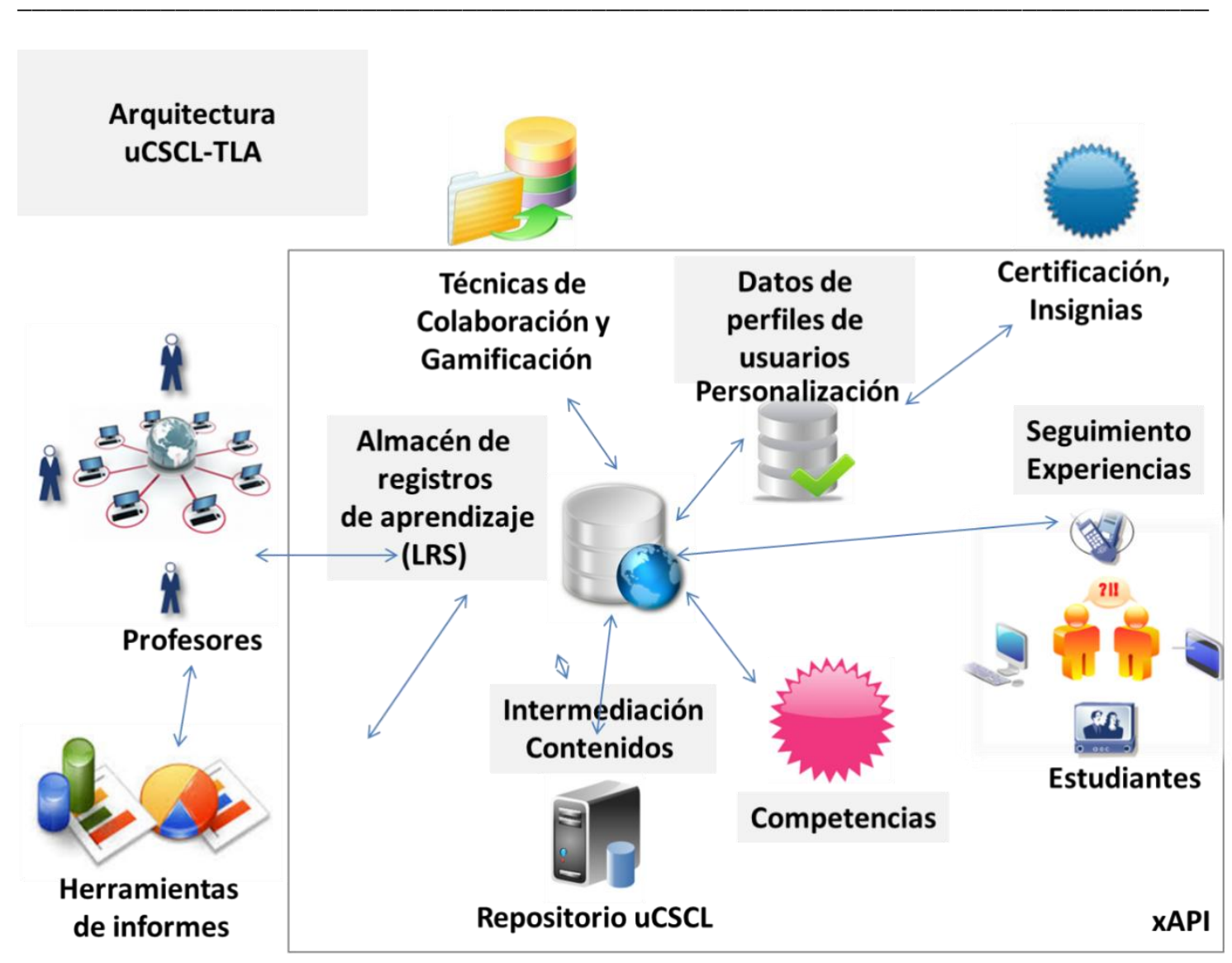

Figura 1. Modelo tecnológico de un MOOC colaborativo basado en la Arquitectura de Formación y Aprendizaje (TLA)

En cuanto a las actividades, nuestro modelo incluye un mecanismo para el diseño de actividades de aprendizaje colaborativo de forma que se garantice que los alumnos trabajan cooperativamente para completar las tareas. Este modelo incluye una serie de patrones de diseño que deben seguirse en las actividades educativas (Collazos, González, Gutiérrez \& Guerrero, 2014). Mientras que la evaluación se lleva a cabo de la manera tradicional (evaluación individual) en el modelo estándar MOOC, nuestra propuesta incluye técnicas de evaluación colaborativa. Para incluir contenidos y actividades, es vital para definir y adaptar los repositorios de objetos de aprendizaje(LO) y metadatos para describir y representar nuevas formas de colaboración establecidos en estos objetos de aprendizaje.

\section{Modelo de evaluación}

Es necesario definir una estrategia de evaluación para poder evaluar y medir la experiencia de aprendizaje en un curso MOOC. Siguiendo el modelo tradicional de 4 niveles de Kirkpatrick (2007) para medir el éxito de acciones formativas (Figura 2) vemos que es posible adaptarlo para incluir en estos niveles la evaluación de los aspectos de gamificación y colaboración. 


\section{Nivel 1. Reacción}

- Satisfacción, interés y compromiso de los estudiantes

\section{Nivel 2. Aprendizaje}

- Conocimientos, habilidades, actitudes y nivel de confianza

\section{Nivel 3. Conducta}

- Cambios de conducta, transferencia de los conocimientos, habilidades y actitudes adquiridos

\section{Nivel 4. Resultados}

- Satisfacción de expectativas, resultados esperados de la formación

Figura 2.Los cuatro niveles de evaluación de una experiencia educativa (Kirkpatrick. (2007)

Asimismo, la comprensión y el análisis del proceso de aprendizaje colaborativo requiere de un análisis secuencial de grano fino de la interacción del grupo en el contexto de los objetivos de aprendizaje. Dillenbourg et al. (1999) afirma que las teorías de aprendizaje colaborativo se centraron durante muchos años sobre cómo los individuos trabajan en un grupo, y se han centrado recientemente en el grupo en sí mismo, tratando de establecer cuándo y bajo qué circunstancias el aprendizaje colaborativo es más eficaz que el aprendizaje individual (Johnson y Johnson). En este contexto, ciertas variables independientes se han identificado y estudiado ampliamente, incluyendo el tamaño del grupo, la composición, la naturaleza y los objetivos de la tarea asignada, los medios y canales de comunicación, la interacción entre pares, el sistema de recompensas involucrados y las diferencias de género (Regan, 2013, Collazos et al., 2002). Investigaciones recientes han puesto énfasis en el estudio de los procesos de colaboración y apoyo (Kinash, 2013; Lewir, 2013).

En un curso para miles de estudiantes, comprensiblemente, se ofrece muy poco margen para la tutorización, seguimiento personalizado y contacto entre el profesorado los estudiantes. Este escaso contacto de los estudiantes con el profesorado puede comprometer el aprendizaje en un curso universitario, y su impacto no debe ser subestimado. No obstante, sería razonable esperar en un curso masivo es muy poco probable que los estudiantes esperen un contacto profundo y frecuente con los docentes. Stewart (2013b) sugiere que la masividad de MOOCs descentra el papel del profesor en la experiencia de aprendizaje. Los MOOC podrían entonces centrarse en su enorme potencial para la interacción entre los propios estudiantes, abordando así el segundo principio de la buena práctica, es decir, fomentar la cooperación entre los alumnos. 


\section{8.- Conclusiones}

Los MOOC se han convertido en un modelo de apoyo a los procesos de enseñanzaaprendizaje impensable hasta hace poco tiempo, desafiando leyes de pedagogía, tecnología y financiera. Sin embargo, aún presentan inconvenientes, sobretodo en aspectos de motivación. Una de las razones de dicha falta de motivación es la forma en la cual muchos de éstos MOOC operan, en la cual no se da una verdadera participación entre los estudiantes y en muchos casos los contenidos son desarrollados de forma individual por un profesor o instructor. En este artículo se presenta un modelo que incluye dos aspectos fundamentales: Colaboración y Gamificación, como estrategias para fomentar una mayor participación en los actores del proceso de enseñanzaaprendizaje.

El diseñar un MOOC es un desafío que requiere considerar diversos aspectos, no solo tecnológicos sino metodológicos. El poder diseñar actividades que conlleven a una verdadera colaboración no es una tarea fácil. No solo involucra colocar a un grupo de participantes en torno a una activad en común; se requiere diseñar actividades que permitan que realmente se dé una colaboración. Es por eso que el modelo propuesto plantea una serie de actividades a realizar con el fin de poder fomentar la colaboración, comunicación y participación y trabajo en equipo.

Creemos que en el futuro, los MOOC deberían apoyar más a los modelos transinstitucionales, y los procesos de aprendizaje secuenciados de forma flexible, reuniendo a profesores y alumnos de todo el mundo con filosofías comunes de educación, intereses complementarios y con experiencia en muchos temas relevantes. Estas nuevas formas de MOOC fomentarán la colaboración entre profesores y alumnos abarcando los límites tradicionales y convirtiendo a la educación superior en una educación internacional e inter-institucional, interdisciplinaria.

Presentación del artículo: 29 de diciembre de 2015 Fecha de aprobación: 15 de enero de 2016 Fecha de publicación: 30 de enero de 2016

González, C.S., Collazos, C.A. y García, R. (2016). Desafío en el diseño de MOOCs: incorporación de aspectos para la colaboración y la gamificación. RED. Revista de Educación a Distancia. 48(7). Consultado el (dd/mm/aaaa) en http://www.um.es/ead/red/48/carina_et_al.pdf

\section{Bibliografía}

Belanger, V., \& Thornton, J. (2013), Bioelectricity: A Quantitative Approach - Duke University's First MOOC. Recuperado de: http://hdl.handle.net/10161/6216 
Blake, D. (2013). From MOOC to DOCC: New Directions in Open Online Education. Recuperado de: http://moocs.com/index.php/from-mooc-to-docc-new-directions-inopen-online-education/

Blom, J., Verma, H., Li, N., Skevi, A., \&Dillenbourg, P. (2013). MOOCs are More Social than You Believe. eLearning Papers, 33. Recuperado de http://www.elearningpapers.eu

Borras O., Martínez M. \&Fidalgo A. (2014). Gamification in MOOC: challenges, opportunities and proposals for advancing MOOC model. ACM Proceedings of the Second International Conference on Technological Ecosystems for Enhancing Multiculturality (TEEM '14).Salamanca. Spain. DOI: 10.1145/2669711.2669902.

Chang, J. W., \& Wei, H. Y. (2015). Exploring Engaging Gamification Mechanics in Massive Online Open Courses. ISSN 1436-4522 (online) and 1176-3647 (print). Journal of Educational Technology \& Society.

Collazos, C., et al. (2002). An Influence Factor in the Collaborative Work Process.Proceedings of 4th International Conference on New Educational Environment, ICNEE 2002, Lugano, Suiza. May 2002, pp. 7-10.

Collazos, C., González, C., y García, R. (2014). Computer Supported Collaborative MOOCs: CSCM. En Proceedings of the 2014 Workshop on Interaction Design in Educational Environments, ACM, pp. 28-33, ISBN: 978-1-4503-3034-3.

Collazos C. et al. (2014). Patterns for Monitoring and Evaluating Collaborative Learning Processes in Videogames. Ed. LAP LAMBERT Academic Publishing, ISBN: 978-3-659-53155-2.

Collazos C. et al. (2007). Evaluating Collaborative Learning Processes using Systembased Measurement. Journal of Educational Technology \& Society, 10, 257-274.

Corbi, A., \& Burgos, D. (2014).Review of current student-monitoring techniques used in elearning-focused recommender systems and learning analytics. The Experience API \& LIME model. International Journal of Interactive Multimedia and Artificial Intelligence. Recuperado de

http://www.ijimai.org/journal/sites/default/files/files/2014/09/ijimai20142_7_6_pdf_ 27449.pdf

Coughlan, S., (2013). Harvard plans to boldly go with 'Spocs'. Recuperado en enero 7, 2016, de http://etcjournal.com/2013/09/26/spocs-are-mooc-game-changers/

Chauhan. A. (2014). Massive Open Online Courses (MOOCS): Emerging Trends un Assessment and Accreditation. En: Digital Education Review, 25, 7-17. Recuperado de http://revistes.ub.edu/index.php/der/article/view/11325 en enero 7, 2016.

Cruz-Benito, J. et al. (2015).Extending MOOC ecosystemsusing web services and software architectures. En P. Ponsa, J. A. Román, \& D. Guasch (Eds.), Actas del XVI Congreso Internacional de Interacción Persona-Ordenador. Interacción 2015 
(Vilanova i la Geltrú, Barcelona, España, 7 al 9 de Septiembre de 2015) (pp. 438444).

Daniel J. (2012). Making Sense of MOOCs: Musings in a Maze of Myth, Paradox and Possibility. Journal of Interactive Media in Education. 3, 18-38. doi:10.5334/201218

Del Blanco, A., et al. (2013). E-Learning standards and learning analytics. Can data collection be improved by using standard data models? En 2013 IEEE Global Engineering Education Conference (EDUCON) (pp. 1255-1261). IEEE. doi:10.1109/EduCon.2013.6530268

De-Marcos, L.et al. (2014). An empirical study comparing gamification and social networking on e-learning. Computers \& Education, 75, 82-91.

Dillenbourg P. (1999). What do you mean by collaborative learning?. P. Dillenbourg. Collaborative-learning: Cognitive and Computational Approaches., Oxford: Elsevier, pp.1-19, 1999.

Downes, S. (2013). Connective Knowledge and Open Resources. Recuperado de http://halfanhour.blogspot.co.uk/2013/10/connective-knowledge-and-openresources.html

González, C., Mora, A. \& Toledo, P. (2014). Gamification in intelligent tutoring systems. En Proceedings of the Second International Conference on Technological Ecosystems for Enhancing Multiculturality (TEEM '14). ACM, New York, NY, USA, 221-225. DOI=10.1145/2669711.2669903. Recuperado de http://doi.acm.org/10.1145/2669711.2669903. Pages 221-225

González, C. \& Mora A. (2015). Técnicas de gamificación aplicadas en la docencia de Ingeniería Informática. RevistaReVisión. Vol 8, No 1. Recuperado de http://bit.ly/1PvpeRT

Johnson, D.W., Johnson, R.T., \& Smith, K.A. (2006). Active learning: Cooperation in the college classroom. Edina: Interaction Book Company.

Johnson, R.T., \& Johnson, D.W. (1986). Cooperative learning in the science classroom. Science and children, 24, 31-32. Recuperado de http://bit.ly/1z5tYTh

Kagan, S. (1990). Cooperative Learning for Learners Limited in Language Proficiency. En M. Brubacher, R. Payne, \& K. Rickett (Eds.), Perspectives on Small Group Learning (pp. 202-223). Oakville: Rubicon Publishing Inc.

Kirkpatrick, D.L., \& Kirkpatrick, J.D. (2007). Implementing the Four Levels. San Francisco: Berrett-Koehler Publishers.

Kinash, S. (2013). MOOCing about MOOCs. Education Technology Solutions, 57, 5658. Recuperado de http://bit.ly/1YQw596 
Kolowich, S., \& Newman, J. (2013). The minds behind the MOOCs. The Chronicle of Higher Education. Recuperado de http://chronicle.com/article/The-ProfessorsBehind-the-MOOC/137905/

Larry, C. (2012). MOOCs and Pedagogy: Teacher-Centered, Student-Centered, and Hybrids. Recuperado de http://larrycuban.wordpress.com/2013/02/13/moocs-andpedagogy-part-2/

Lewir, T. (2013). After Setbacks, Online Courses Are Rethought. The New York Times. Recuperado de http://www.nytimes.com/2013/12/11/us/after-setbacks-onlinecourses-are-rethought.html

Liyanagunawardena, T., Williams, S. \& Adams, A. (2013). The Impact and Reach of MOOCs: A Developing Countries' Perspective. eLearning Papers, 33. Recuperado de http://www.elearningpapers.eu

McAuley, A. et al. (2010). The MOOC model for digital practice. Recuperado de: http://davecormier.com/edblog/wp-content/uploads/MOOC_Final.pdf

Morrison, D. (2013). How Collaborative Learning Works in Closed Online Courses vs. MOOCs. Online Learning Insight Blog. Recuperado de: http://onlinelearninginsights.wordpress.com/2013/02/20/how-does-collaborativelearning-work-in-closed-online-courses-vs-moocs/

Pappano, L., (2012). The Year of the MOOC. The New York Times. Retrieved from http://www.nytimes.com/2012/11/04/education/edlife/massive-open-online-coursesare-multiplying-at-a-rapid-pace.html

Poltrack J. et al. (2012). The Next Generation of SCORM: Innovation for the Global Force. Paper presented at the Interservice/Industry Training, Simulation \& Education Conference (I/ITSEC).

Regan, D. (2013). The Training and Learning Architecture: Infrastructure for the Future of Learning. XV Simposio Internacional de Tecnologías de la Información y las Comunicaciones en la Educación (SINTICE 2013).

Reigeluth, C. (2012). Teoría instruccional y tecnología para el nuevo paradigma de la educación. RED, Revista de Educación a Distancia. Número 32. 30 de septiembre de 2012.Consultado el 16/05/13 en http://www.um.es/ead/red/32

Romero, M. \& Usart, M. (2013).Serious Games Integration in an Entrepreneurship Massive Online Open Course (MOOC). In Serious Games Development and Applications (pp. 212-225). Heidelberg: Springer Berlin.

Shah, D. (2015). MOOCs in 2015: Breaking Down the Numbers. Consultado el 28/12/2015 en: https://www.edsurge.com/news/2015-12-28-moocs-in-2015breaking-down-the-numbers. 
Soller, A. (2001). Supporting social interaction in an intelligent collaborative learning system. International Journal of Artificial Intelligence in Education (IJAIED), 12, 40-62. Recuperado de: https://halshs.archives-ouvertes.fr/hal-00197321/

Stahl, G., Koschmann, T., \&Suthers, D. (2006). Computer-supported collaborative learning: An historical perspective. In R.K. Sawyer (Ed.), Cambridge handbook of the learning sciences (pp. 409-426). Cambridge, UK: Cambridge University Press.

Steenkamp, A. \& González C. (2011).Special Issue on Service-oriented Software Engineering: Foundations, Architectures, SDLCs and Innovative Applications. International Journal of Information Technologies and the Systems Approach (IJITSA). Recuperado de: http://www.igi-global.com/Files/Ancillary/1935570X_4_1_Preface2.pdf

Totten, S., et al. (1991). Cooperative learning: A guide to Research. New York: Garland.

Wenger, E. (2009). A social theory of learning. In K. Illeris (Ed.), Contemporary theories of learning (pp. 209-218). New York: Routledge.

Werbach, K. (2012). For the Win: How Game Thinking Can Revolutionize Your Business. Wharton: Wharton Digital Press.

Yuan, L., \& Powell S. (2013). MOOCs and Open Education: Implications for Higher Education. Retrievedfrom: http://publications.cetis.ac.uk/2013/667

Zapata-Ros, M. (2014) Los MOOC en la crisis de la Educación Universitaria. Docencia, diseñoyaprendizaje. Amazon.

Zichermann, G., \& Cunningham, C. (2011). Gamification by Design: Implementing Game Mechanics in Web and Mobile Apps. Sebastopol, California: O'Reilly Media.

\section{Agradecimientos}

Este trabajo ha sido parcialmente financiado por el Proyecto de Investigación CYTED, ref. 513RT0481 "Red Iberoamericana de Apoyo a los Procesos de EnseñanzaAprendizaje de Competencias Profesionales a través de Entornos Ubicuos y Colaborativos (U-CSCL)" y al proyecto español InDAGuS (TIN2012-37826-C02). 